\title{
Derivation and Characterization of Recombinants of the Lr54/Yr37 Translocation in Common Wheat
}

\author{
Ian Heyns ${ }^{1}$, Zacharias Pretorius ${ }^{2}$ and Francois Marais ${ }^{1, *}$ \\ ${ }^{1}$ Department of Genetics, University of Stellenbosch, Private Bag X1, Matieland 7602, Stellenbosch, South Africa \\ ${ }^{2}$ Department of Plant Sciences, University of the Free State, PO Box 339, Bloemfontein 9300, South Africa
}

\begin{abstract}
The wild relatives are an important source of new genes for the genetic improvement of wheat. Leaf and stripe rust resistance genes Lr54 and Yr37 occur on an Aegilops kotschyi-derived chromosomal translocation that had apparently replaced wheat chromosome arm $2 \mathrm{DL}$. The alien chromatin also includes the locus of a gene for reduced plant height $(H)$, which appears to be different from $R h t 8$ on chromosome arm 2DS. The introgressed genes were mapped relative to homoeologous wheat marker loci following the induction of chromosome pairing in translocation heterozygotes that lacked the Ph1 locus. Ten recombined Lr54/Yr37 translocation chromosomes were derived and characterized with microsatellite, AFLP and SCAR markers. The data suggested that there was significant homoeology between the full-length translocated segment and the wheat 2DL chromosome arm. The recombined translocations apparently resulted from single crossovers during which the distal end of the long arm of the translocation chromosome was replaced with wheat chromatin. Recombinant (Lr54/Yr37-74) retained the least alien chromatin and both resistance genes, yet had lost the reduced plant height gene. A polymorphic AFLP fragment was converted into a dominant SCAR marker to detect rec. \#74. In addition three wheat microsatellite loci that map to the introgressed region provide a useful recessive marker system to detect $L r 54 / Y r 37$. The shortened translocation could be useful in breeding and may be used for continued, closer mapping of the resistance genes.
\end{abstract}

Keywords: Chromosome engineering; leaf rust resistance; $P h 1$; stripe rust resistance, Triticum aestivum L.

\section{INTRODUCTION}

Leaf rust $($ Puccinia triticina $=P$. recondita Roberge ex Desmaz. f. sp. tritici) and stripe rust $(P$. striiformis f. sp. tritici Westend) resistance genes Lr54 and Yr37 were transferred to common wheat (Triticum aestivum L.) from Aegilops kotschyi Boiss. [1]. The translocation derives from a plant that was double monosomic for 2D and an unknown Ae. kotschyi group 2 chromosome and it was probably formed following centric breaking and subsequent fusion of an Ae. kotschyi chromosome $2 \mathrm{~L}$ arm with $2 \mathrm{DS}$ of wheat. The stripe rust resistance proved to be effective against prevailing pathotypes in South Africa and Australia [2], whereas Lr54 provided effective resistance against all the South African and western Canadian P. triticina pathotypes [1]. The introgression appeared to include an uncharacterized gene for reduced plant height that could limit its use in breeding. The translocated Ae. kotschyi chromosome arm also failed to pair with its common wheat 2DL homoeologue during meiosis causing the alien genes to be inherited as a single, large linkage block. Such linkage blocks can be broken up by inducing synapsis and crossing over between the alien and homoeologous wheat chromosome segments [3-6]. Even though homoeologous crossover frequencies are considerably reduced compared to homologous crossover frequencies, and

*Address correspondence to this author at the Department of Plant Sciences, Loftsgard Hall, NDSU Dept 7670, PO Box 6050, Fargo, ND 58108-6050, USA; Tel: 701-231-7973; Fax: 701-231-8474; E-mail: ansie@ hotmail.com also vary with the evolutionary divergence of the wheat and alien chromatin [7], the methodology simultaneously allows for genetic/physical mapping of the introgressed region [8]. The use of an appropriate wheat nulli tetrasomic or ditelosomic stock $[3,9]$ as the testcross parent produces testcross progeny that are hemizygous for the chromosome region involved in the translocation. As a result, both wheat specific and alien chromatin specific genetic markers can be employed for analysis of the testcross progeny. Well developed and publicly available wheat genetic maps [10-12] provide adequate markers that are evenly spread across the region affected by the introgression. The extent and distribution of recombination will reflect the degree of homoeology and compensating ability of the translocation. Chromosome structural differences that occur within otherwise homoeologous regions may, however, cause aberrant meiotic pairing structures and formation of single crossover products that appear to result from complex multiple crossovers $[8,13]$. Interpretation of these products may be difficult yet could provide an indication of the type of structural difference that was involved. Such information could help with deciding which recombination products are most likely to be useful. Chromosome structural differences that are encountered may be the result of evolutionary divergence of the alien and corresponding common wheat chromosome regions [14]; may be introduced through the involvement of the Chinese Spring homoeologous pairing locus deletion line (CSphlb) which is chromosomally unstable [15]; or it may be due to natural karyotype variation within wheat itself [16-18]. 
This study aimed, firstly, to confirm the presence of a height reducing gene (here designated $H$ ) on the $L r 54 / Y r 37$ translocation. Secondly, homoeologous pairing was induced between the translocated chromosome region and the normal wheat 2DL chromosome arm (through deletion of the Phl locus) and the progeny analyzed to find recombinants for the translocated region. The recombinants were used as indicator of homoeology between the foreign and wheat DNA; to determine the relative locations of the $L r 54, \operatorname{Yr} 37$ and $H$ loci and to identify recombinants in which the height reducing gene had been separated from the resistance genes.

\section{MATERIALS AND METHODOLOGY}

\section{Plant Material and Seedling Resistance Screening}

Line CS-Lr54/Yr37 (pedigree = CS monosomic 2D// Ae. kotschyi-617/ $4 * \mathrm{CS} / 3 / \mathrm{CS}-\mathrm{S}$ ) was the source material used in the study; it is a near-isogenic line of the common wheat, Chinese Spring (CS) and carries a 2DL translocation from Ae. kotschyi. In the above pedigree, parent CS-S (pedigree = Inia $66 / 7 * C S$ ) is a semi-dwarf near-isogenic line of CS that has acquired the reduced height gene, $R h t-B 1 b$ (from Inia 66) on chromosome $4 \mathrm{BS}$.

The presence of Lr54 in segregating populations was detected by employing seedling leaf rust resistance tests. This was done with the use of Puccinia triticina pathotype UVPrt8 which is virulent on the common wheat genotypes used in the study (CS, CS-S and W84-17) yet is avirulent on Lr54 (infection type = ;1). Line W84-17 is a semi-dwarf spring wheat (pedigree 'Inia 66'/5/ 'El Gaucho'/ Son 64/4/ $\mathrm{Tg} / 3 /$ Son 64// Tzpp/ Nai 60). Seedling screening tests to detect $\operatorname{Yr} 37$ relied on the use of $P$. striiformis $\mathrm{f}$. sp. tritici pathotype 6E22A-. The wheat background genotypes are susceptible to pathotype 6E22A- whereas $\operatorname{Yr} 37$ provides resistance (infection type $; \mathrm{c}-; 1^{\mathrm{n}}$ ). Seedling resistance tests were done as described earlier [1].

\section{Confirmation of a Gene for Reduced Height on the Lr54 Translocation}

The effect of the translocation on plant height was investigated by making two sets of crosses. Firstly, CS (tall phenotype; Rht-BlaRht-Bla hh) was crossed with W84-17 (semi-dwarf phenotype; Rht-B1bRht-B1b $h h$ ). The $\mathrm{F}_{1}$ was backcrossed reciprocally to the parents and $F_{2}$ seeds were harvested. The material served as control populations to provide an indication of the variation in height resulting from the segregation of Rht genes in the CS and W84-17 backgrounds. Secondly, experimental populations segregating for the Lr54 translocation were produced. CS was crossed with resistant (UVPrt8) plants selected from the W84-17 near-isogenic line $\mathrm{F}_{1}: 0514$ (CS-Lr54-Yr37/4*W8417; Rht-B1bRht-B1bHh). Leaf rust resistant $\mathrm{F}_{1}$ plants were selected and backcrossed to the parents. The parents, $F_{1}$ and $\mathrm{F}_{2}$ progeny together with the respective backcrosses of both groups were raised in a greenhouse. As it was necessary to screen the plants in the experimental group for the presence of $L r 54$, all the plants in both the experimental and control groups were subjected to leaf rust seedling tests and were then transplanted to pots in a greenhouse. In the greenhouse, four plants were grown per $2 \mathrm{~L}$ plastic pot and seedlings were randomly allocated to pots and positions within pots. The pots were randomly placed in a water-cooled greenhouse with automated irrigation. Commercially available nutrient mixtures were applied following the manufacturer's guidelines. At maturity, plant height $(\mathrm{cm})$ was measured from the base of the plant to the tip of the ear on the primary spike. Standard errors, confidence intervals and Student's t-test statistic (one-tailed) were calculated (Microsoft Excel) to compare the mean plant heights of populations.

\section{Derivation and Characterization of Translocation Recombinants}

The CS-Lr54/Yr37 translocation line was crossed with, and the $\mathrm{F}_{1}$ backcrossed to, the CSphlb mutant. The $\mathrm{F}_{1}$ plants were screened for seedling resistance $(L r 54)$ to $P$. triticina pathotype UVPrt8 and absence of the Phl locus (using a PCR marker [19]). Plants that were phlb deletion homozygotes and at the same time heterozygous for Lr54 were selected and testcrossed with the leaf rust susceptible breeding line, W84-17. A total of $468 \mathrm{TF}_{1}$ (04M144) seeds were produced (this will be referred to as the primary testcross population). Initially, an attempt was made to characterize the primary testcross population with the use of chromosome 2DL restriction fragment length polymorphism loci. However, when suitable markers of this type could not be found it was decided to use wheat chromosome 2DL microsatellite markers instead. In order to apply the microsatellite markers (present in wheat but absent in Ae. kotschyi chromatin) it was necessary to first derive testcross plants monosomic for the translocated chromosome 2D. To achieve this, resistant $\mathrm{TF}_{1}$ : 04M144 plants were used to pollinate CS nullisomic 2D tetrasomic 2A (CSN2DT2A) or CS nullisomic 2D tetrasomic 2B (CSN2DT2B) plants. DNA extracts [20] were made of the resistant progeny from the crosses (secondary testcross population) and used for microsatellite analysis.

Eight 2DL microsatellite markers (loci Xbarc228, Xwmc41, Xcfd233, Xgwm539, Xgwm157, Xcfd50, Xwmc167 and $X g d m 6$ ) and four 2DS microsatellite markers (loci Xbarc124, Xgwm261, Xgwm484 and Xcfd116) were used in the study. Microsatellite amplification products were obtained and visualized by silver staining [21, 22]. Relevant map detail and primer sequences were obtained from the Graingenes website [23]. The microsatellite markers were confirmed to be specific for the respective chromosome arms and to be polymorphic (present in CS and W84-17 but absent within the Lr54 translocated chromosome region). For this purpose they were tested on a genotype panel consisting of: Ae. kotschyi accession 617, CS, W84-17, CSN2AT2B, CSN2BT2A, CSN2DT2A, CS ditelosomic 2DS (CSDT2DS), CSDT2DL and a hemizygote for the segment of translocated Ae. kotshyi chromatin (= CS-Lr54/Yr37 hemizygote; $2 \mathrm{n}=41$ $+\mathrm{t}=$ monotelodisomic 2DS plus 2D $\left.\mathrm{D}^{\text {Lr54/Yr37 translocation }}\right)$.

\section{Further Characterization of the Translocation Recombi- nants}

When allosyndetic recombinants were identified, these were characterized with additional sequence characterized amplified region (SCAR) and amplified fragment length polymorphism (AFLP) markers. The SCAR markers included primer set Ust2b [24] which amplifies a 2DL wheat- 
specific locus and primer set Sopw7 which amplifies a fragment of the same length in several alien translocations yet not in wheat [8].

The genotype panel that was used to confirm the microsatellite loci was also used in a search for polymorphic AFLP markers associated with 2DL. Fluorescence-based semi-automated AFLP analysis was done as outlined in the 'AFLP Plant Mapping Kit' of PE/Applied Biosystems Inc. (850 Lincoln Centre Drive, Foster City, CA 94404, USA). The final amplification step utilized five MseI selective primers (-CAT, -CTC, -CAG, -CTA and -CTG) which were used in all possible combinations with fluorescently labelled EcoRI-ACA (6-FAM $\left.{ }^{\mathrm{TM}}\right)$, EcoRI-AAC $\left(\mathrm{NED}^{\mathrm{TM}}\right)$ and EcoRI- AGG (JOE $\left.{ }^{\mathrm{TM}}\right)$ selective primers. The amplification products were detected with an Applied Biosystems 3130XL Genetic Analyzer (Foster City, California, USA) operated by the Central Analytical Facility (http://academic.sun.ac.za/saf/) at Stellenbosch University. "GeneMapper" software [25] was used to visualize and analyze the results.

\section{Development of a Dominant Marker}

An AFLP polymorphism that was specific for the shortest recombinant was converted into a SCAR marker. Following unlabeled selective AFLP amplification, the polymerase chain reaction (PCR) product was size separated on a $6 \%$ $(\mathrm{w} / \mathrm{v})$ denaturing poly-acrylamide sequencing gel and silver stained. The region containing the polymorphic band was excised from the Ae. kotschyi and CS (negative control) lanes; the DNA was eluted with a GenElute gel extraction kit (Sigma-Aldrich Chemie Gmbh, Munich, Germany), reamplified and separated on $2 \%$ agarose gel. Methodology and kits from Promega Corporation (2800 Woods Hollow Road, Madison, WI 53711-5399, USA) were then used to transform and clone the fragment. Clones were sequenced at the Central Analytical Facility of Stellenbosch University. Sequence analysis was done using the program BioEdit [26] and primers were designed using OligoAnalyzer [27].

The newly designed primers were evaluated on the ten translocation recombinants as well as panels of resistant and susceptible $F_{2}(20)$ and $F_{3}(31)$ plants derived from the first backcross of the shortest recombinant to W84-17. The PCR reaction mixture consisted of $200 \mathrm{ng}$ of template DNA, $1 \mathrm{X}$ PCR buffer, $1.5 \mathrm{mM} \mathrm{MgCl}_{2}, 0.2 \mathrm{mM}$ of each dNTP, $0.5 \mu \mathrm{M}$ of each primer, $1 \mathrm{U}$ of Taq DNA polymerase and $\mathrm{dH}_{2} \mathrm{O}$ to a final volume of $25 \mu \mathrm{l}$. A thermal cycler (Applied Biosystems) was programmed as follows: $5 \mathrm{~min}$ at $94^{\circ} \mathrm{C}, 35$ cycles consisting of $1 \mathrm{~min}$ at $94^{\circ} \mathrm{C}, 1 \mathrm{~min}$ at $61^{\circ} \mathrm{C}$ and $1 \mathrm{~min}$ at $72^{\circ} \mathrm{C}$ followed by a final extension step at $72^{\circ} \mathrm{C}$ for $7 \mathrm{~min}$ and a soak temperature of $4^{\circ} \mathrm{C}$. Following PCR amplification the PCR product was size separated on a $1.5 \%$ agarose gel (Ethidium bromide, $1 \mathrm{X}$ Tris/Borate/EDTA) together with a molecular size marker.

\section{RESULTS AND DISCUSSION}

\section{Gene for Reduced Plant Height}

The distribution of plant height measurements in the parental lines, $F_{1}$, backcross and $F_{2}$ populations of the control and experimental groups of crosses are shown in Fig. 1. The parental lines, 0514, W84-17 and CS, formed distinct height groups (95\% confidence level) with average heights of $71 \pm$
$6.9 \mathrm{~cm}($ Rht-B1bRht-B1b Hh), $95 \pm 2.0 \mathrm{~cm}(R h t-B 1 b R h t-B 1 b$ $h h)$ and $149 \pm 3.9 \mathrm{~cm}($ Rht-BlaRht-Bla hh), respectively.

The height data of the control progeny reflected the segregation of the single, major height reducing gene, Rht-Blb. The $\mathrm{F}_{1}($ Rht-B1aRht-B1b hh) distribution was similar to that of CS confirming that the Rht-Bla allele is dominant. The height distributions of the $F_{2}$ and the two $B_{1}$ populations fell within the ranges spanned by the parental lines. With the exception of one backcross plant, the plants in all the groups were taller than $80 \mathrm{~cm}$. Only one backcross plant was taller than $180 \mathrm{~cm}$. The average height of $F_{1}$ plants in the experimental group was eight centimetres less than in the control group. The experimental group showed much wider distributions of individual plant heights for both backcrosses and the $\mathrm{F}_{2}$. As in the control group, plants were never taller than 180 $\mathrm{cm}$; however, many plants were shorter than $80 \mathrm{~cm}$. For backcrosses to $\mathrm{CS}$, the mean height of the experimental group $(134 \mathrm{~cm})$ was lower $(\mathrm{P}=0.016)$ than that of the control group $(145 \mathrm{~cm})$. A comparable difference $(\mathrm{P}<0.0001)$ was seen for backcrosses to the shorter parent. In the latter, the mean height of the experimental group was $72 \mathrm{~cm}$ compared to $104 \mathrm{~cm}$ in the control group. With respect to the $F_{2}$, t-test comparison showed that the experimental group $(110 \mathrm{~cm})$ was on average significantly $(\mathrm{P}<0.0001)$ shorter than the control group $(131 \mathrm{~cm})$. Similarly, resistant $F_{2}$ plants within the experimental group (average height $=106.6 \mathrm{~cm}$ ) were significantly shorter than the susceptible plants (average height $=128.9 \mathrm{~cm})(\mathrm{P}<0.01)$; thus confirming the presence of a height-reducing gene. The shorter stature $(71 \mathrm{~cm})$ of the resistant, near-isogenic $0514 \mathrm{~F}_{1}$ plants (Rht-B1bRht-B1b Hh) as compared to the recurrent parent, W84-17 $(95 \mathrm{~cm}$; Rht$B 1 b R h t-B 1 b h h)(\mathrm{P}<0.01)$ and that of the experimental group $\mathrm{F}_{1}(138 \mathrm{~cm} ;$ Rht-BlaRht-B1b Hh) as compared to the control $\mathrm{F}_{1}(146 \mathrm{~cm} ;$ Rht-BlaRht-B1b hh $)(\mathrm{P}=0.087)$ suggested that $H$ is a dominant gene. Furthermore, the height reducing effect of $H$ was much less pronounced in the presence of Rht-Bla, ie $H$ is hypostatic to $R h t-B 1 a$.

The height reducing genes $R h t 4, R h t 7$ and $R h t 8$ occur on homoeologous group 2 chromosomes and produce semidwarf phenotypes. Unlike the GA-insensitive $R h t-B 1 b$ and $R h t-D 1 b$ genes they do not affect coleoptile and early plant growth [28]. Gene Rht7 (chromosome 2A) was induced through EMS treatment and is associated with a detrimental effect on yield [29]. Rht4 is another induced mutation that occurs on 2BL [30]. Only Rht8 has been widely used in breeding. It was derived from the Japanese landrace Akakomugi in the 1920s and was mapped close to the microsatellite locus, Xgwm261 on 2DS [30,31]. The dwarfing gene, $H$, described here is associated with $2 \mathrm{DL}$ and its relationship (or not) with the group $2 R h t$ genes is unclear. While $H$ has not been evaluated for its potential use in breeding, it will be useful to have recombinants of the $L r 54 / Y r 37$ translocation with and without the height reducing gene.

\section{Identification of $\mathrm{Lr} 54 / \mathrm{Yr} 37$ Translocation Recombinants}

One hundred and nine resistant plants from the primary testcross population were used to pollinate CSN2DT2A or CSN2DT2B plants. The $\mathrm{F}_{1}$ (hemizygous $2 \mathrm{D}$ ) of each secondary testcross was seedling screened for the presence of $\operatorname{Lr} 54$ and a resistant plant from each population was used for DNA extraction and microsatellite analysis. Based on the micro- 


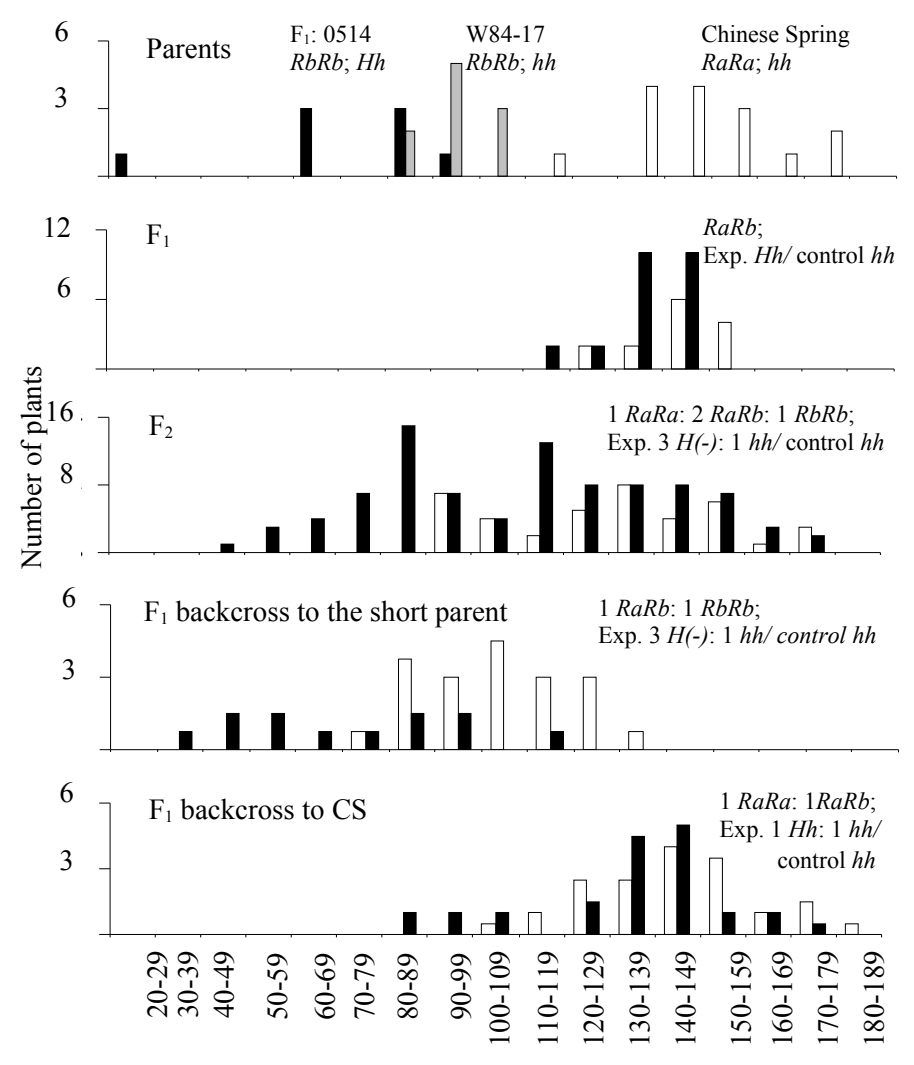

Plant height $(\mathrm{cm})$

Fig. (1). Plant height distributions in the parental lines, $F_{1}, F_{2}$ and backcross generations of a control cross (W84-17 X CS) and an experimental cross involving the $L r 54 / Y r 37$ translocation ( $\mathrm{F}_{1}$ : 0514 X CS). Colour codes used for the parental lines are white (CS), grey (W84-17) and black $\left(\mathrm{F}_{1}: 0514=\mathrm{W} 84-17-L r 54 / Y r 37\right)$. Progeny distributions in the control group are shown with white bars and that in the experimental group with black bars. Also indicated are the respective height genotypes with $R a=R h t-B 1 a ; R b=R h t-B 1 b$ and $H=$ unknown height reducing gene associated with the Lr54/Yr37 translocation.

satellite data, ten translocation recombinants could be identified, the results of which are shown in Table 1 . The order of the eight chromosome arm 2DL microsatellite loci [12] is shown in Table 1; however, the relative location of $\mathrm{Xgdm} 6$ with respect to $X w m c 167$ is uncertain. Conventional linkage analysis was not done in view of the relatively small number of recombinants that were obtained. Additional, unmapped AFLP and SCAR loci were subsequently employed for the continued characterization of the ten recombinants and were ordered relative to the mapped loci by following a physical mapping approach (Table 1). Six polymorphic AFLP loci were discovered following an initial (duplicate) screen of the control genotypes and ten recombinants. The productive selective primer pairs and the sizes of the polymorphic PCR fragments are given in Table 1. Each locus could be amplified in Ae. kotschyi and the Lr54/Yr37 translocation yet was absent in the wheat parents. The results were confirmed on a further independent panel of $16 \mathrm{~B}_{1} \mathrm{~F}_{1}$ (CS-Lr54/Yr37 translocation $/ 2 * \mathrm{CSph} 1 b$ mutant) plants, eight with (resistant) and eight without (susceptible) the Lr54/Yr37 translocation.

The recombined translocation chromosomes that are described in Table $\mathbf{1}$ are the results of two meiotic events: Firstly, in the absence of $P h l$ crossover occurred between the alien segment and 2D of the CSphlb line to produce the ten primary wheat-alien recombined chromosomes. A normal, second meiosis then occurred in the primary testcross $F_{1}$ which involved each recombined translocation chromosome and 2D of the testcross parent (W84-17).

\section{Homoeologous Recombination with 2DL}

The molecular marker data of Table 1 reveal the following: (1) Allosyndetic recombination between the CSLr54/Yr37 translocated segment and chromosome arm 2DL of CSphlb (Fig. 2A) produced ten recombinants which were resolved into three size categories by the marker loci. In the formation of each recombinant, crossover occurred proximally of the $X c f d 50$ locus and CSphlb chromatin replaced a distal region of alien chromatin. The modified primary translocations will have acquired CSph1b 2DL telomeres and all the wheat microsatellite loci that were restored would have expressed the CS-specific alleles (Fig. 2A). (2) The primary recombined wheat-alien 2DL arms then paired and recombined regularly with 2DL of W84-17 during the second meiosis. This follows from the fact that the W84-17 allele of locus $X c f d 50$ occurred in nine of the ten secondary recombinants. (3) Each of recombinants \#74 and \#265 expressed both the W84-17 and CS alleles of Xcfd50 (but only one of the two Xgdm6 alleles) and therefore contained duplicated regions. The duplications were most likely introduced during 
Table 1. Data Obtained Following the Characterization of Ten Allosyndetic Recombinants of the CS-Lr54/Yr37 Translocation and Controls with Molecular Markers. Symbols: "S" and "W" Respectively Indicate Absence or Presence of Wheat Specific Marker Fragments Associated with the Microsatellite and SCAR Xust2-IIJ ${ }_{1}^{d}$ loci. In the Case of the AFLP, SCAR 410 and SCAR Xsopw7 loci, which Amplify Ae. Kotschyi-Specific Fragments, "S" and "W" Respectively Indicate Presence and Absence of the Alien Marker Fragments. Symbol ".." Indicates Chromosome Regions that Appear to be Deleted

\begin{tabular}{|c|c|c|c|c|c|c|c|c|c|c|c|c|c|c|}
\hline & 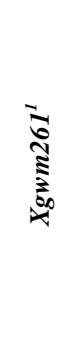 & 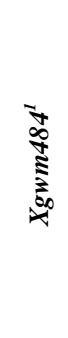 & 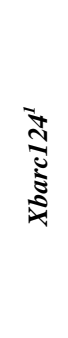 & 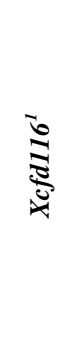 & 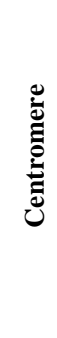 & $\begin{array}{c}\text { Xbarc228 } \\
\text { Xwmc41 } \\
\text { Xcfd233 } \\
\text { AFLP }^{2} \\
-410 \\
\text { Lr54 } \\
\text { Yr37 }\end{array}$ & 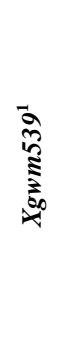 & 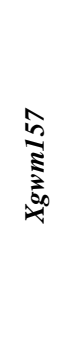 & $\begin{array}{c}\text { AFLP }^{2} \\
-306 \\
-360 \\
-416 \\
\text { SCAR Xust2-IIJ }{ }_{I}^{d} \\
\text { SCAR Xsopw } 7\end{array}$ & $\begin{array}{c}\text { AFLP }^{2} \\
-212 \\
-228\end{array}$ & $\frac{\bar{Z}}{5}$ & 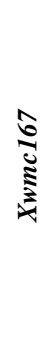 & 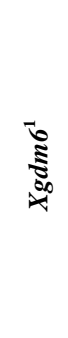 & 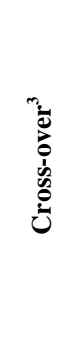 \\
\hline & \multicolumn{4}{|c|}{ 2DS } & & \multicolumn{9}{|c|}{ 2DL } \\
\hline Ae. kotschyi & $S$ & $\mathrm{~S}$ & $\mathrm{~S}$ & $S$ & & $S$ & $S$ & $S$ & $S$ & $S$ & $\mathrm{~S}^{*}$ & $S$ & $S$ & \\
\hline Lr54/Yr37-hemizygote & $\mathrm{W}^{\mathrm{CS}}$ & $\mathrm{W}^{\mathrm{CS}}$ & $\mathrm{W}^{\mathrm{CS}}$ & $\mathrm{W}^{\mathrm{CS}}$ & & $S$ & $S$ & $S$ & S & $S$ & S & $S$ & $S$ & \\
\hline $\mathrm{CS}$ & $\mathrm{W}^{\mathrm{CS}}$ & $\mathrm{W}^{\mathrm{CS}}$ & $\mathrm{W}^{\mathrm{CS}}$ & $\mathrm{W}^{\mathrm{CS}}$ & & $\mathrm{W}$ & $\mathrm{W}^{\mathrm{CS}}$ & $\mathrm{W}$ & $\mathrm{W}$ & $\mathrm{W}$ & $\mathrm{W}^{\mathrm{CS}}$ & $\mathrm{W}$ & $\mathrm{W}^{\mathrm{CS}}$ & \\
\hline Recombinant \#37 & $\mathrm{W}^{\mathrm{W}}$ & $\mathrm{W}^{\mathrm{W}}$ & $\mathrm{W}^{\mathrm{W}}$ & $\mathrm{W}^{\mathrm{CS}}$ & & $S$ & $S$ & $S$ & $S$ & $\mathrm{~W}$ & $\mathrm{~W}^{\mathrm{W}}$ & $\mathrm{W}$ & $\mathrm{W}^{\mathrm{W}}$ & (i) \\
\hline Recombinant \#205 & $\mathrm{W}^{\mathrm{CS}}$ & $\mathrm{W}^{\mathrm{CS}}$ & $\mathrm{W}^{\mathrm{CS}}$ & $\mathrm{W}^{\mathrm{CS}}$ & & $S$ & $S$ & $S$ & $S$ & $\mathrm{~W}$ & $\mathrm{~W}^{\mathrm{W}}$ & $\mathrm{W}$ & - & (ii) \\
\hline Recombinant \#256 & $\mathrm{W}^{\mathrm{W}}$ & $\mathrm{W}^{\mathrm{W}}$ & $\mathrm{W}^{\mathrm{W}}$ & $\mathrm{W}^{\mathrm{CS}}$ & & $S$ & S & $S$ & $S$ & $S$ & $\mathrm{~W}^{\mathrm{CS}}$ & $\mathrm{W}$ & $\mathrm{W}^{\mathrm{CS}}$ & (iv) \\
\hline Recombinant \#265 & $\mathrm{W}^{\mathrm{CS}}$ & $\mathrm{W}^{\mathrm{CS}}$ & $\mathrm{W}^{\mathrm{CS}}$ & $\mathrm{W}^{\mathrm{CS}}$ & & S & $S$ & $S$ & $S$ & $S$ & $\mathrm{~W}^{\mathrm{CS} \& \mathrm{~W}}$ & $\mathrm{~W}$ & $\mathrm{~W}^{\mathrm{CS}}$ & (v) \\
\hline Recombinant \#119 & $\mathrm{W}^{\mathrm{W}}$ & $\mathrm{W}^{\mathrm{W}}$ & $\mathrm{W}^{\mathrm{CS}}$ & $\mathrm{W}^{\mathrm{CS}}$ & & S & S & $\mathrm{S}$ & $S$ & $S$ & $\mathrm{~W}^{\mathrm{W}}$ & $\mathrm{W}$ & - & (ii) \\
\hline Recombinant \#148 & $\mathrm{W}^{\mathrm{W}}$ & $\mathrm{W}^{\mathrm{CS}}$ & $\mathrm{W}^{\mathrm{CS}}$ & $\mathrm{W}^{\mathrm{CS}}$ & & $S$ & S & $\mathrm{S}$ & $S$ & $S$ & $\mathrm{~W}^{\mathrm{W}}$ & $\mathrm{W}$ & - & (ii) \\
\hline Recombinant \#247 & $\mathrm{W}^{\mathrm{W}}$ & $\mathrm{W}^{\mathrm{CS}}$ & $\mathrm{W}^{\mathrm{CS}}$ & $\mathrm{W}^{\mathrm{CS}}$ & & $S$ & S & $S$ & $S$ & $S$ & $\mathrm{~W}^{\mathrm{W}}$ & $\mathrm{W}$ & - & (ii) \\
\hline
\end{tabular}

${ }^{1}$ These microsatellite markers produced distinct CS and W84-17 alleles which are indicated with the superscripts CS and W, respectively. Superscripts CS \& W indicate duplicated loci.

${ }^{2}$ Primer combinations that detected the respective AFLP loci (fragment size in brackets): EcoRI-AGG/MseI-CAG (212 bp); EcoRI-AGG/MseI-CAG (228 bp); EcoRI-AAC/MseICAT (410 bp); EcoRI-ACA/MseI-CTG (306 bp), EcoRI-AGG/MseI-CTG (360 bp); EcoRI-AAC/MseI-CTG (416 bp).

${ }^{3}$ Possible meiotic pairing structure and position on 2DL where crossover could have occurred (Fig. 2) to produce the particular recombinant.

the second meiosis as a result of a structural (intrachromosomal translocation?) difference between the CSphlb and W84-17 sub-telomeric regions. (4) Six recombinants appeared to carry a deletion of the $\mathrm{Xgdm6}$ locus that probably resulted from the same aberration. Subsequent backcrossing of rec. \#74 to W84-17 also resulted in segregates (Table 2) that had the Xgdm6 locus deleted and thus confirmed the presence of the chromosome structural difference.

The second meiosis occurred in testcross $F_{1}$ plants that carried a primary recombined wheat-alien translocation chromosome plus a complete chromosome 2D from W84-17 (Fig. 2B). As will be shown below, the data of Tables 1 \& 2 imply that (compared to the sequence in CSphlb and published map [12]) the relative locations of Xcfd50 and Xgdm6 are switched in W84-17 chromosome 2D. Such a difference could have arisen following an intra chromosome arm translocation of either locus. The precise nature and extent of the structural difference cannot be deduced from the limited data; however, the recombination products obtained are consistent with a translocation rather than a duplicated or inverted region.

Inter-variety chromosome structural differences appear to occur frequently in common wheat. Non-homologous, reciprocal translocations were detected (mostly through meiotic analyses) in studies involving both wheat varieties and species [17]. Yasumuro and co-workers found that multivalent configurations that are typical of reciprocal translocation differences occurred in $81 \%$ of $466 \mathrm{~F}_{1}$ cross combinations. Furthermore, sixty two of 117 Japanese common wheat land races and varieties differed from CS by 1-2 reciprocal translocations involving at least three different pairs of chromosomes [32]. C-band studies [18] revealed translocations, deletions, duplications and inversions within a large and diverse collection (499 accessions) of tetraploid and hexaploid wheat as well as triticale. One hundred and fourty of these had structurally different chromosomes. Certain aberrations 

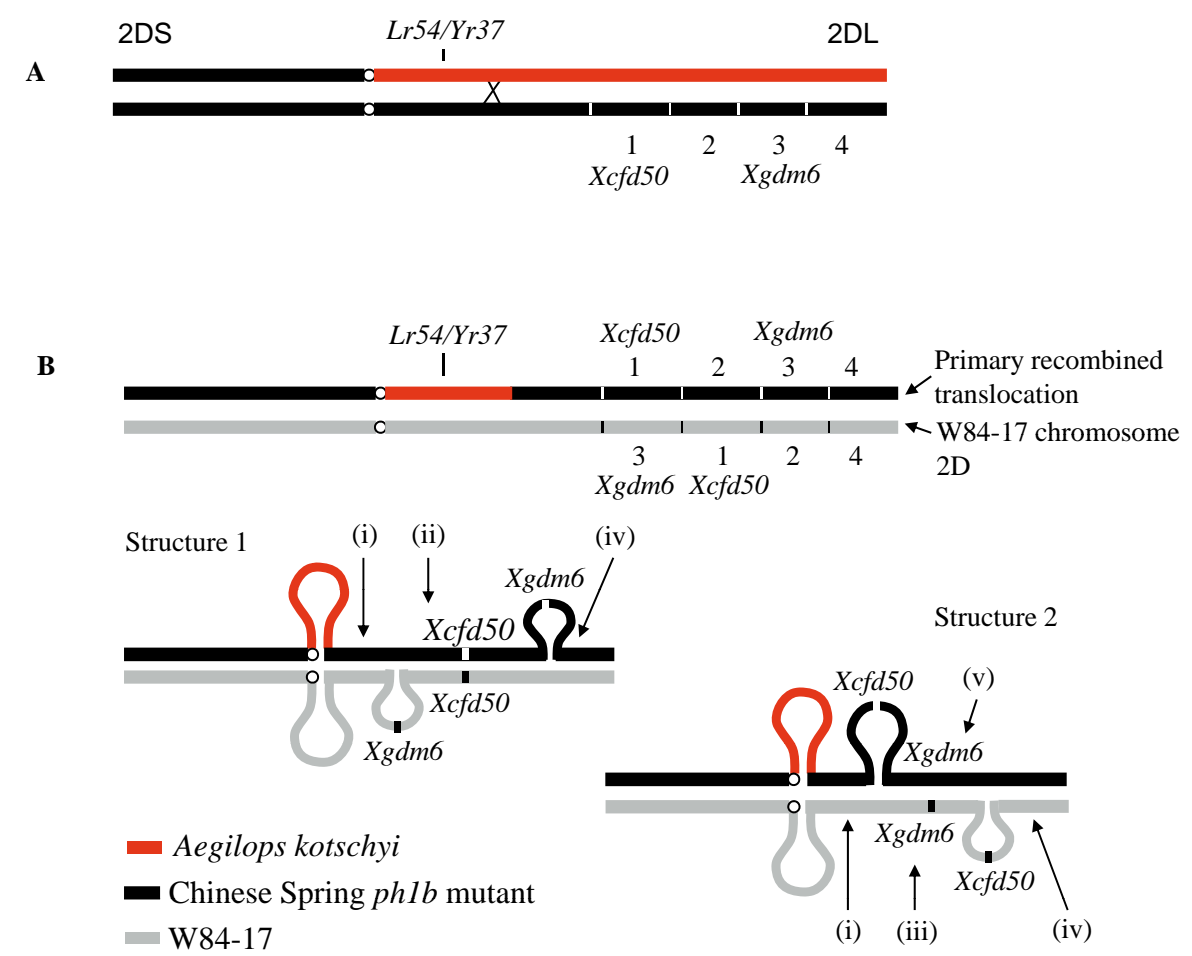

Fig. (2). Sketches showing the probable origin of ten secondary recombined translocation chromosomes of which the marker data are summarized in Table 1. (A) During a first (allosyndetic) meiosis, 2DL of CSphlb recombined with the homoeologous Ae. kotschyi-derived chromosome segment to produce the respective primary translocation recombinants. (B) During the second meiosis (Ph1 present) the translocated chromosomes 2DL recombined with 2DL of W84-17 which has a structurally different sub-telomeric region. In the 2DL arm of W84-17, a chromosome region surrounding Xgdm6 occurs proximally, rather than distally from Xcfd50. This structural difference results in two possible aberrant pairing structures being formed. Crossover within the two pairing configurations may result in formation of normal gametes, gametes carrying an Xcfd50 duplication or an Xgdm6 deletion.

were widespread while others occurred less frequently. While C-banding proved useful for the detection of large aberrations, smaller structural differences or those involving chromosome regions with few bands would have remained undetected. The latter can only be reliably exposed by employing more laborious genetic and aneuploid mapping procedures [18].

Table 2. Results Obtained Following Screening for the Presence of Microsatellite loci Xgdm6 and Xcfd50 in $15 \mathrm{BC}_{1} \mathrm{~F}_{2}$ : Recombinant \#74/2* W84-17 Plants

\begin{tabular}{|l|l|l|}
\hline Number of $\mathrm{BC}_{1} \mathrm{~F}_{2}$ Plants: & Xgdm6 & Xcfd50 \\
\hline Eight resistant & $\mathrm{W}^{\mathrm{W}}$ & $\mathrm{W}^{\mathrm{W}}$ \\
\hline One resistant & - & $\mathrm{W}^{\mathrm{W}}$ \\
\hline Five susceptible & $\mathrm{W}^{\mathrm{w}}$ & $\mathrm{W}^{\mathrm{W}}$ \\
\hline One susceptible & - & $\mathrm{W}^{\mathrm{W}}$ \\
\hline & & \\
\hline
\end{tabular}

Heterozygosity for the Xcfd50 - Xgdm6 structural difference resulted in aberrant pairing during the second meiosis. Possible meiotic pairing structures that could have been produced are shown in Fig. (2B). The relative positions within the pairing structures where crossovers could have occurred to produce the respective secondary recombinant chromosomes are shown in Fig. (2B) and given in Table 1. Recombinant \#74 was subsequently used in backcrosses to W84-17.
Since recombinant chromosome \#74 carried an $X c f d 50$ duplication (\#74 (dupl Xcfd50)-Xgdm6 $)$; aberrant pairing structures (containing compensation loops) could once again form in plants that were either heterozygous or homozygous for this specific translocation. Crossing over within such structures could have resulted in translocation chromosomes without Xgdm6 (\#74 ${ }^{\text {Xcfd50-(del Xgdm6) }}$ ). Characterization of $15 \mathrm{BC}_{1} \mathrm{~F}_{2}$ plants for the presence of microsatellite loci $X c f d 50$ and $X g d m 6$ (Table 2) showed that two of the plants did in fact lack $X g d m 6$. Thus, in a cross involving genotypes that differ structurally for the $X c f d 50-X g d m 6$ region, rec. \#74 progeny may be one of four types, i.e. \#74 ${ }^{\text {Xcfd50-Xgdm6 }}, \# 74^{\text {Xgdm6-Xcfd50, }}$ $\# 74^{X c f d 50 \text {-(del } X g d m 6)}$ or \#74 (dupl Xcfd50)-Xgdm6 . During subsequent inbreeding a percentage of the homozygous derivatives could therefore be agronomically inferior. However, as nine allosyndetic crossovers occurred between the translocation \#74 breakpoint and the Xcfd50/Xgdm6 loci (Table 1), there is sufficient genetic recombination distance to readily recover plants that combine the resistance with either of the Xcfd50Xgdm6 sequences.

The ten primary allosyndetic recombinants apparently resulted from single crossovers involving the Ae. kotschyi and homoeologous wheat $(\mathrm{CSph} 1 b)$ chromatin. Although limited in extent, the data suggest that significant homoeology existed between the two regions, however, the observed allosyndetic recombination frequency $(=0.092)$ between Lr54 and Xcfd50/Xgdm6 is still low as could be expected [7]. The recombinants appear to have regained functional 2DL telomeres and probably restored significant portions of the 
wheat loci on this arm. The allosyndetic crossover that produced rec. \#74 occurred within the most proximal of three chromosome arm 2DL deletion bins [12] (Table 1). Only $25 \%$ of 185 EST-loci on 2DL were found to occur within the most proximal deletion bin, which accounts for $49 \%$ of the chromosome arm length [33]. This would suggest that more than $75 \%$ of the $2 \mathrm{DL}$ common wheat loci could have been restored in rec. \#74.

\section{Recombination with 2DS}

Marker loci Xgwm261, Xgwm484 and Xcfd116 were mapped to the most proximal deletion bin on chromosome arm 2DS whereas Xbarc124 was mapped to the most distal deletion bin [12]. The full-length translocation and primary recombinants would have expressed the CS allele of each locus. During the second meiosis, crossover occurred between the CS and W84-17 derived 2DS chromosome arms. Since recombination frequencies increase towards the telomeres, it can be expected that in the secondary recombinants, CS alleles would predominate towards the centromere (and alien chromatin) whereas W84-17 alleles would predominate towards the telomere. This was used as basis for ordering the four loci on chromosome arm 2DS (Table 1) and suggested that Xbarc124 may actually have a more proximal location, in-between $\mathrm{Xgwm} 484$ and $X c f d 116$. Furthermore, three of the markers detected null alleles in rec. \#74 which would suggest the presence of a large deletion distally from Xcfd116. Depending on its size and the genes involved, it could detrimentally affect plant phenotype. Since the aberration is also distant from the alien introgressed region, it should be possible to restore a complete 2DS arm through homologous crossover. This can for example be achieved between a rec. \#74 and a rec. \#37 chromosome. Tertiary recombinant progeny of the desired type can then be selected with the use of marker loci Xsopw 7 (specific for the longer translocation \#37) and Xgwm484 (occurs within the deleted area).

Thus, although a high frequency of deletions and duplications occurred among the secondary recombinants, these were brought about by chromosome structural differences inherent to the wheat parental genotypes, and involved chromosome regions that are well-removed from the introgressed alien chromatin regions. These aberrations should not impact on the utility of the translocation.

\section{Recombinant \#74}

In Rht-Blb homozygotes the added presence of gene $H$ resulted in a dwarf phenotype. When resistant secondary $\mathrm{TF}_{1}, \mathrm{TF}_{2}$ and $\mathrm{TF}_{3}$ progeny (segregated for Rht-Bla and Rht$B 1 b)$ of the ten recombinant lines were evaluated for plant height, the progeny of four recombinants (\#119, \#205, \#247 and \#256) included dwarfs, which suggested that they had retained $H$. The progeny of the shortest recombinant (\#74) did not include any dwarfs. To confirm that it had lost $H$, resistant (20 plants) and susceptible (11 plants) $\mathrm{BCF}_{3}$ (rec. \#74/ 2*W84-17) progeny were evaluated for plant height. When the average plant height of the susceptible and resistant groups was compared, the resistant group was only two $\mathrm{cm}$ shorter, whereas the distribution of plant heights was similar for the two groups. This suggested that $H$ is no longer associated with rec. \#74 and allowed for determining its approximate map location (Table 1). Seedling inoculation with
P. striiformis pathotype 6E22A- showed that $Y r 37$ had been retained in each of the ten recombinants. Near-isogenic lines of rec. \#74 are being developed in adapted varieties in order to fully evaluate the utility of the Lr54/Yr37 genes.

\section{Marker Development}

One of the six AFLP loci (detected with EcoRIAAC/MseI-CAT (410bp)) was retained in the shortest recombinant (\#74) and was used as basis for deriving a SCAR marker. The critical band was excised from the Ae. kotschyi lane, purified and cloned. A clone with the correct insert size was identified and sequenced. From the sequence, forward (5': ACCAATTCAACTTGCCAAGAG) and reverse (5': GAGTAACATGCAGAAAACGACA) primers were derived and validated on the ten recombinant and parental lines as well as on two additional panels of resistant and susceptible $\mathrm{BCF}_{2}$ and $\mathrm{BCF}_{3}$ (respectively, 20 and 31 plants derived from the cross: Rec. \#74/2* W84-17) segregating for the presence of recombinant chromosome \#74. The new primer set consistently amplified a $410 \mathrm{bp}$ band in Ae. kotschyi, the Lr54/Yr37 translocation lines and resistant $\mathrm{BCF}_{2}$ and $\mathrm{BCF}_{3}$ plants but not in susceptible control plants.

In addition to the newly-developed, dominant SCAR marker of rec. \#74, it can also be detected using wheat microsatellite loci. Since the Lr54/Yr37-74 recombined translocation chromosome has null-alleles for the microsatellite loci Xbarc228, Xwmc41 and Xcfd233, the latter may be used as recessive haplotype markers for its detection. For each of the three marker loci, the polymorphisms can readily be observed by separating the PCR product on $2 \%$ agarose gels, thus providing a rapid and inexpensive assay. As null alleles may also occur in normal wheat germplasm, simultaneous testing for the presence of two, or all three of the loci in a multiplex PCR reaction, should provide for more accurate labelling of rec. \#74. When used in conjunction with the dominant SCAR S14-74 marker, it will therefore be possible to distinguish translocation homo- and heterozygotes.

\section{ACKNOWLEDGEMENT}

We wish to thank the Winter Cereal Trust and National Research Foundation (South Africa) for funding the project.

\section{REFERENCES}

[1] Marais GF, McCallum B, Snyman JE, Pretorius ZA, Marais AS. Leaf rust and stripe resistance genes Lr54 and $Y r 37$ transferred to wheat from Aegilops kotschyi. Plant Breeding 2005; 124:538-41.

[2] Marais GF, Pretorius ZA, Marais AS, Wellings CR. Transfer of rust resistance genes from Triticum species to common wheat. S Afr J Plant Soil 2003; 20: 193-98.

[3] Sears ER. In: Evans LT, Peacock WJ, Eds. Transfer of alien genetic material to wheat. Wheat Science - today and tomorrow. Cambridge University Press, London 1981; 75-89.

[4] Knott DR. The wheat rusts - breeding for resistance, SpringerVerlag, New York 1989; pp. 201.

[5] Jauhar PP, Chibbar RN. Chromosome-mediated and direct gene transfers in wheat. Genome 1999; 42: 570-83.

[6] Qi L, Friebe B, Zhang P, Gill BS. Homoeologous recombination, chromosome engineering and crop improvement. Chromosome Res 2007; 15: 3-19.

[7] Lukaszewski AJ. Physical distribution of translocation breakpoints in homoeologous recombinants induced by the absence of the Phl gene in wheat and triticale. Theor Appl Genet 1995; 90: 71419.

[8] Marais GF, Badenhorst PE, Eksteen A, Pretorius ZA. Reduction of Aegilops sharonensis chromatin associated with resistance genes Lr56 and Yr38 in wheat. Euphytica 2010; 171: 15-22. 
[9] Sears ER, Sears LMS. In: Ramanujam S, Ed. The telocentric chromosomes of common wheat. Proc. $5^{\text {th }}$ Int. Wheat Gen. Symp. New Delhi 1979; 389-407.

[10] Röder MS, Korzun V, Wendehake K, et al. A microsatellite map of wheat. Genetics 1998; 149: 2007-23.

[11] Somers DJ, Isaac P, Edwards K. A high-density microsatellite consensus map for bread wheat (Triticum aestivum L.). Theor Appl Genet 2004; 109: 1105-14.

[12] Sourdille P, Singh S, Cadalen T, et al. Microsatellite-based deletion bin system for the establishment of genetic-physical map relationships in wheat (Triticum aestivum L). Funct Integr Genomics 2004; 4: 12-25.

[13] Marais GF, Kotze L, Eksteen A. Allosyndetic recombinants of the Aegilops peregrina-derived Lr59 translocation in common wheat. Plant Breeding 2010; 129: 356-61.

[14] Levin DA. The role of chromosomal change in plant evolution. Oxford series in ecology and evolution, Oxford University Press, NY. 2002; pp. 230.

[15] Sanchez-Moran E, Benavente E, Orellana J. Analysis of karyotype stability of homoeologous-pairing $(p h)$ mutants in allopolyploid wheats. Chromosoma 2001; 110: 371-77.

[16] Vega C, Fominaya A, Ferrer A. Influence of chromosome structure on the degree of meiotic pairing of intercultivar wheat hybrids (Triticum aestivum L.). Heredity 1987; 58: 357-63.

[17] Schlegel R. A compendium of reciprocal translocations in wheat: 2nd Edition. Wheat Inf Serv 1996; 83: 35-46.

[18] Badaeva ED, Dedkova OS, Gay G, et al. Chromosomal rearrangements in wheat: their types and distribution. Genome 2007; 50: 907-26.

[19] Gill KS, Gill BS. A PCR-based screening assay of $P h 1$, the chromosome pairing regulator gene of wheat. Crop Sci 1996; 36: 71922.

[20] Doyle JJ, Doyle JL. Isolation of plant DNA from fresh tissue. Focus 1990; 12: 13-15.

[21] Groenewald JZ, Marais AS, Marais GF. Amplified fragment length polymorphism-derived microsatellite sequence linked to the Pch1 and Ep-D1 loci in common wheat. Plant Breeding 2003; 122: 83-85.

[22] Beidler JL, Hilliard PR, Rill RL, Ultrasensitive staining of nucleic acids with silver. Anal Biochem 1982; 126: 374-380.

[23] Graingenes: A database for Triticeae and Avena [Homepage on the internet]. Agricultural research service of the US department of agriculture [cited 2009 July 25]. Available from: http://wheat.pw. usda.gov/GG2/index.shtml/

[24] Marais GF, Groenewald CW, Marais AS. SCAR markers that specifically tag chromosomes $2 \mathrm{D}$ of wheat and $2 \mathrm{~J}_{1}{ }^{\mathrm{d}}$ of Thinopyrum distichum. S Afr J Plant Soil 2009; 26: 129-31.

[25] GeneMapper ${ }^{\circledR}$ Software [Homepage on the Internet]. Applied Biosystems - Life Technologies Corporation; c2010 [cited 2009 November 15]. Available from: https://products.appliedbiosystems. com/ab/en/US/adirect/ab?cmd=catNavigate $2 \&$ catID $=600798 \&$ tab $=$ DetailInfo/

[26] BioEdit v 7.0.5 [Homepage on the internet]. Hall T: Ibis Therapeutics, 1891 Rutherford Road, Carlsbad CA; c1997-2005 [updated 2005 May 31; cited 2009 November 20]. Available from: http:// www.mbio.ncsu.edu/BioEdit/bioedit.html/

[27] Oligo Analyzer [Homepage on the internet]. Hitachi Software Engineering America, Ltd. c2007-2008 [cited 2009 November 20]. Available from: http://smartnote.miraibio.com/bioinformatics/ oligo_analyzer_ebi?PHPSESSID=a94f7375ba7a609fd1a83e12d29 $043 \mathrm{c} \overline{3}$

[28] Ellis MH, Rebetzke GJ, Chandler P, Bonnett D, Spielmeyer W, Richards RA. The effect of different height reducing genes on the early growth of wheat. Funct Plant Biol 2004; 31: 583-89.

[29] Worland AJ, Law CN, Shakoor A. The genetical analysis of an induced height mutant in wheat. Heredity 1980; 45: 61-71.

[30] Ellis MH, Rebetzke GJ, Azanza A, Richards RA, Spielmeyer W. Molecular mapping of gibberellin-responsive dwarfing genes in bread wheat. Theor Appl Genet 2005; 111: 423-30.

[31] Korzun V, Röder MS, Ganal MW, Worland AJ, Law CN. Genetic analysis of the dwarfing gene $(R h t 8)$ in wheat. Part I. Molecular mapping of Rht8 on the short arm of chromosome 2D of bread wheat (Triticum aestivum L.). Theor Appl Genet 1998; 96: 110409.

[32] Yasumuro Y, Ali AM, Nakata N, Tomita M. In: Slinkard AE, Ed. Unexpected high frequency of reciprocal translocation exists in Japanese common wheat cultivars. Proc. $9^{\text {th }}$ Int. Wheat Genet. Symp. Saskatoon, Saskatchewan 1998; 122-24.

[33] Conley EJ, Nduati V, Gonzalez- Hernandez JL, et al. A 2600-locus chromosome bin map of wheat homoeologous group 2 reveals interstitial gene-rich islands and co-linearity with rice. Genetics 2004; 168: 625-37. 